\title{
Stressbewältigung
}

\section{Oft ganzheitliche Behandlung gewünscht}

- "Es ist nicht entscheidend was wir erleben, sondern wie wir es erleben", betonte Dr. Tobias Sprenger, Köln. Eine akute Stressreaktion ist physiologisch sinnvoll, weil sie durch die Ausschüttung von Katecholaminen ( $z$. B. Adrenalin) eine rasche, kurzfristige Leistungssteigerung des Körpers bewirkt, damit dieser angemessen reagiert.

Chronische Stressreaktionen, die mit einem erhöhten Cortisolspiegel einhergehen, können jedoch gesundheitsschädliche Konsequenzen haben: seelische Beschwerden, Schlafstörungen, oft auch Herz-Kreislauf-Erkrankungen, erhöhtes Schlaganfallrisiko, Stoffwechselprobleme mit Magenoder Reizdarmsymptomen, verstärkte Infektanfälligkeit, sogar Krebserkrankungen bis hin zum „Burn-out-Syndrom“.

Jeder Mensch empfindet die Grenze zwischen Anregung und Bedrohung durch einen äußeren Stressfaktor unterschiedlich. Die Mehrzahl der Stressbetroffenen wünscht sich allerdings eine ganzheitliche
Behandlung, so Sprenger. Neurodoron ${ }^{\circledR}$ ist ein anthroposophisches Arzneimittel, das Gestressten helfen kann, ihre gesunde Mitte und einen harmonischen Lebensrhythmus wiederzufinden. Die drei mineralischen Inhaltsstoffe Gold, EisensulfatQuarz und Kaliumphosphat sollen harmonisierend auf Rhythmusstörungen sowie normalisierend und entlastend auf das Nerven-Sinnes-System wirken. Sprenger konnte mit diesem Ansatz bereits vielen Stresspatienten helfen. Die gute Verträglichkeit, das Fehlen von Kontraindikationen, die Kombinierbarkeit mit konventionellen oder alternativen Therapien wie z. B. Autogenem Training oder Heileurythmie und der schnelle Wirkeintritt, z. T. bereits nach einem Tag, haben sich in seiner täglichen Praxis vielfach bewährt.

Die Anthroposophische Medizin versteht sich nicht als Alternative, sondern als Ergänzung zur Schulmedizin. Rudolf Steiner, Begründer der Anthroposophie, baute

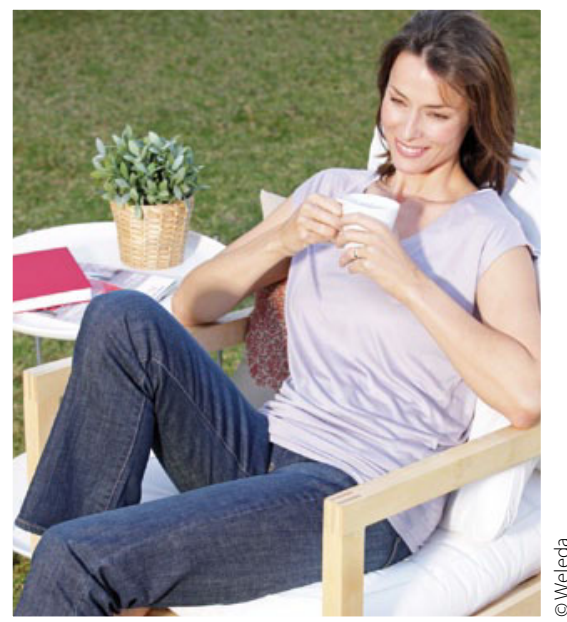

Mit Unterstützung anthroposophischer Medizin einen gesunden Lebensrhythmus wiederfinden.

vor 90 Jahre das Unternehmen Weleda auf, das seitdem ökologisch und nachhaltig verschiedene anthroposophische Gesundheitspräparate produziert.

- Dr. Ulrike Fortmüller

Quelle: Presseworkshop „90 Jahre Weleda Arzneimittel", Lana/Südtirol, Juni 2011 (Veranstalter: Weleda)

\section{Chronische wässrige Diarrhöen \\ Denken Sie auch an eine mikroskopische Kolitis!}

- Wird bei chronischen wässrigen Diarrhöen endoskopisch kein krankhafter Befund erhoben, wird häufig die Diagnose „Reizdarm“ gestellt. „Doch bei solchen Patienten müssen Sie immer an das Vorliegen einer mikroskopischen Kolitis denken", sagte Priv.-Doz. Dr. Ahmed Madisch, Hannover. Um dieses Krankheitsbild zu diagnostizieren, müssen immer Stufenbiopsien entnommen werden.

Bei der mikroskopischen Kolitis, zu der die lymphozytäre und kollagene Kolitis gehören, handelt es sich letztendlich um eine chronisch-entzündliche Darmerkrankung. „Sie ist häufiger als man denkt", so Madisch. Bei ca. 10\% aller Patienten mit chronischen nicht blutigen Diarrhöen könne diese Diagnose gestellt werden, bei über 70-Jährigen sogar in über $20 \%$ der Fälle.

Das klinische Bild ist charakterisiert durch wässrige Diarrhöen, die auch nachts auftreten, häufig verbunden mit krampfartigen abdominellen Schmerzen, Übelkeit, Gewichtsverlust und Anämie. Das Krankheitsbild führt zu einer deutlichen Einschränkung der Lebensqualität.

\section{Evidenzbasierte Therapie}

Bis vor einigen Jahren stand für die mikroskopische Kolitis keine evidenzbasierte Therapie zur Verfügung. Erst in den letzten Jahren wurde in drei placebokontrollierten Studien die Wirksamkeit von Budesonid (Budenofalk ${ }^{\circledR}$ ) in einer Dosierung von $9 \mathrm{mg} / \mathrm{Tag}$ belegt. „Nach diesen Ergebnissen handelt es sich bei Budesonid um ein hoch effektives Therapieprinzip; denn die Ansprechrate liegt bei über $80 \%$ ", so Madisch. Entsprechend liege die Number needed to treat nur bei zwei Patienten.

Bei einem Großteil der primär erfolgreich behandelten Patienten entwickelt sich jedoch ein Rezidiv, das eine Langzeittherapie erforderlich macht. In zwei placebokontrollierter Doppelblindstudien wurde die Effektivität von Budesonid $(6 \mathrm{mg}$ täglich) gegenüber Placebo über sechs Monate bei kollagener Kolitis untersucht. Die Remissionsraten betrugen knapp $90 \%$.

Angesichts dieser Studienergebnisse empfiehlt Madisch, die Therapie mit $9 \mathrm{mg}$ Budesonid täglich zu beginnen. Nach einer Erhaltungstherapie mit $6 \mathrm{mg}$ über sechs bis acht Wochen sollte das Medikament abgesetzt werden. Bei Auftreten eines Rezidivs sollte die Therapie mit 9 mg Budesonid entweder intermittierend oder kontinuierlich fortgeführt werden.

\footnotetext{
- Dr. med. Peter Stiefelhagen

Quelle: Pressegespräch im Rahmen des DGIM-Kongresses, Wiesbaden, April 2011 (Veranstalter: Dr. Falk Pharma GmbH)
} 